\title{
Shadowing effects on the microstructure of obliquely deposited films
}

\author{
Paritosh \\ Princeton Materials Institute and Department of Mechanical and Aerospace Engineering, \\ Princeton University, Princeton, New Jersey 08544 and Department of Chemical Engineering, \\ University of Michigan, Ann Arbor, Michigan 48109 \\ D. J. Srolovitz \\ Princeton Materials Institute and Department of Mechanical and Aerospace Engineering, \\ Princeton University, Princeton, New Jersey 08544
}

(Received 17 April 2001; accepted for publication 7 November 2001)

Two spatial dimension front tracking simulations have been performed to study the growth of polycrystalline, faceted films from randomly oriented nuclei by varying the deposition angle of the incident flux during physical vapor deposition. The orientation of grain columns, the porosity, the crystallographic texture, and grain size are sensitive to the deposition angle. The origin of this effect is widely believed to be associated with shadowing. In order to isolate the effects of shadowing from other physical effects (such as surface diffusion, deposition species size, flux divergence, etc.), we have constructed a simulation where all of these effects are completely removed. These simulations demonstrate that while many of the observed structural properties of obliquely deposited films are controlled by shadowing, a few key properties cannot be attributed solely to shadowing. (C) 2002 American Institute of Physics. [DOI: 10.1063/1.1432125]

\section{INTRODUCTION}

Designing novel techniques for the deposition of thin films with special structures is of significant interest. One method to control the film microstructure is to deposit the film using a collimated vapor flux of atoms and varying the angle of incidence of the flux with respect to substrate normal. ${ }^{1,2}$ The film microstructure depends sensitively on this angle of incidence. Obliquely deposited films exhibit a unique columnar structure. ${ }^{3}$ If the flux is incident on the substrate from an angle that is large with respect to the film normal, the columns are commonly surrounded by a network of voids. The material within the column can be either a single grain or polycrystalline. Columnar microstructures typically lead to film with anisotropic electrical, ${ }^{4}$ optical, ${ }^{5}$ and magnetic properties, ${ }^{6}$ and nonrandom crystallographic texture. ${ }^{7}$ At low growth temperatures (i.e., $T<0.5 T_{m}$, where $T_{m}$ is the melting temperature), these columns tend to tilt away from the surface normal, toward the incoming flux, as shown in Fig. 1.

Several empirical relationships were proposed to account for this variation of the column angle $\beta$ with the flux angle $\alpha$ (see Fig. 1) based on a large set of experimental data on amorphous and crystalline films. ${ }^{3,8}$ Nieuwenhuizen and Haanstra $^{8}$ were the first to report a systematic set of microstructure observations of column orientation based on microfractographs of aluminum films. Based on their observations, they proposed the following empirical relationship:

$\tan \beta=\frac{1}{2} \tan \alpha$

which is often referred to as the "tangent law." This relationship provides a reasonable description of the microstructure for the range of incidence angles $0^{\circ} \leqslant \alpha \leqslant 60^{\circ} .^{3,9}$ Based on a continuum model, Lichter et al. ${ }^{10}$ derived a theoretical form for the relationship between column orientation and deposition angle,

$$
\tan \beta=\frac{2}{3}\left(\frac{\tan \alpha}{1+\psi \tan \alpha \sin \alpha}\right),
$$

where $\psi$ is a function of the diffusion length, the vapor flux, and the amplitude of the initial surface profile. Based on geometrical arguments, Tait et al., ${ }^{11}$ derived another expression relating $\beta$ to $\alpha$ for the case of limited surface diffusion,

$$
\beta=\alpha-\sin ^{-1}\left[\frac{1-\cos \alpha}{2}\right] .
$$

In addition to increasing the column angle $\beta$, increasing the flux angle $\alpha$ also leads to increased porosity. ${ }^{3,12,13}$

There have been many theoretical and simulation models for the structure of obliquely deposited films. Since microstructures of interest are typically on a scale much larger than interatomic spacings, analysis based on continuum models ${ }^{10,14}$ is appropriate and have lead to some important insights. On the other hand, inclusion of such important features as the polycrystalline nature of the film, nonlocal shadowing, and faceting are difficult to incorporate into continuum models. Many atomic scale computer simulations of film growth been performed, using such methods as Monte Carlo, ${ }^{15}$ molecular dynamics, ${ }^{16,17}$ and ballistic deposition models. ${ }^{3,18}$ Interestingly, it has been argued that the formation of realistic columnar structures requires the inclusion of the discreteness (finite size) of the depositing species, which is explicitly missing from the continuum models.

In this paper, we employ a continuum growth model to determine the role of shadowing in determining the microstructure of obliquely deposited films. Our approach explicitly includes nonlocal shadowing, a polycrystalline film, and 

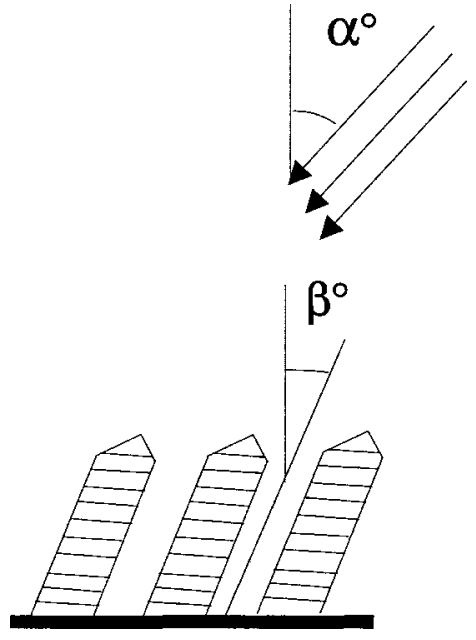

FIG. 1. Schematic showing the oblique deposition geometry, where $\alpha$ is the angle the incident vapor flux makes with respect to the substrate normal and $\beta$ is the mean column angle.

crystal anisotropy (faceting), but includes neither finite size deposition species, surface diffusion or preferred orientations of nuclei. The goal of this work is to separate the effects of shadowing from those of surface diffusion, discreteness or preferential nucleation, which are also thought to play a major role in determining the microstructure of obliquely deposited films. The present simulation method is based upon our earlier model for faceted polycrystalline film growth. ${ }^{19,20}$ We examine the microstructures produced as a function of flux angle $\alpha$, crystal symmetry, and attachment kinetics.

\section{SIMULATION METHOD}

The simulation algorithm employed in this study is based upon the principal of evolutionary selection, as suggested by van der Drift. ${ }^{21}$ Paritosh et al. ${ }^{20}$ have discussed a simulation algorithm that accounts for evolutionary selection in the growth of polycrystalline films. The model is based of an array of faceted crystallites in which each facet grows normal to itself. All of the crystal nuclei form simultaneously on the substrate (i.e., no renucleation during growth) in random locations and with random orientations at the beginning of the simulation. Experimentally, random nucleation seldom occurs in film growth (even on amorphous substrates) owing to the anisotropy of surface and interfacial energies and atomic transport. While nucleation of islands with some preferred orientation with respect to the substrate is often observed in experiment, such effects were explicitly neglected here such that the effects of shadowing during growth could be easily discerned, separate from other effects. Preferential nucleation would clearly bias the evolution of the crystallographic texture and grain size. The simulations do model the precoalescence (i.e., island growth) stage of film growth, however, this paper focuses mainly on the postcoalescence period of film growth. The present model also does not explicitly include diffusional interactions between grains or islands. This does not necessarily conflict with the assumption that the grains are faceted, since faceting readily occurs in film growth processes where surface diffusion is negligible, such as in diamond film growth or in cases where the sticking coefficient is anisotropic. The focus of the present study is on shadowing effects and, therefore, explicitly ignores phenomena associated with diffusional interactions between facets, grains or islands.

In the present model, the growth velocity of each facet depends upon the flux it receives

$$
v=\hat{n} \cdot \mathbf{J},
$$

where $v$ is the velocity of the facet, $\hat{n}$ is the facet normal, and $\mathbf{J}$ is a vector parallel to the flux direction and with the units of velocity (i.e., the product of the flux and an atomic volume). The dynamics of the surface is local, such that the velocity of each segment of the surface depends on the flux it receives. A natural consequence of this, is that when vertices, where two facets meet, disappears (is subsumed during growth) it will never reappear. We note that while this is in line with expectations (since a surface that is buried well never again receive any flux and grow), it is not reproduce by the simulations performed by some other groups (see, e.g., Ref. 22), which allow grains to grow through one another. In the present simulations, we track both grain boundaries and free surfaces. A grain boundary is formed when two differently oriented crystallites touch and the grain boundary extends as the point of contact moves (i.e., as the surfaces of the two grains meeting at the boundary continue to grow). The grain boundary location is, therefore, simply a trace of the point on the surface where two grains intersect (there is no grain boundary migration in this model). No grain boundary migration is considered in the present simulations. New surface facets may form during growth (i.e., a grain boundary can split into two surfaces).

The simulation tracks the motion of each vertex. In the present two-dimensional model a vertex is:

(1) An intersection between two facets of a single crystalline grain;

(2) A point where two grains meet; or

(3) A point of intersection of part of a facet that receives flux and part that is shadowed.

The vertex velocity (speed and direction) are calculated from the velocities and orientations of the bounding facets. While the facet orientations are fixed by the crystallographic orientation of the grain, the positions and existence of individual vertices will change during the simulation. The simulation proceeds by integrating the equations of motion of the vertices forward in time. The time step used for the finite difference solution of the equations of motion for the vertices is variable and is chosen to be the time required for the next vertex intersection in the entire system. Intersection times are precalculated for each vertex pair. When an intersection does occur, the facet between the intersecting vertices is removed and the velocity and orientation of the new vertex formed is determined from the orientations of the new neighboring facets.

Several types of shadowing are considered in these simulations. Shadowing arises when (see Fig. 2):

(1) One or more facets of a grain prevents other facets of the same grain from receiving flux; and 


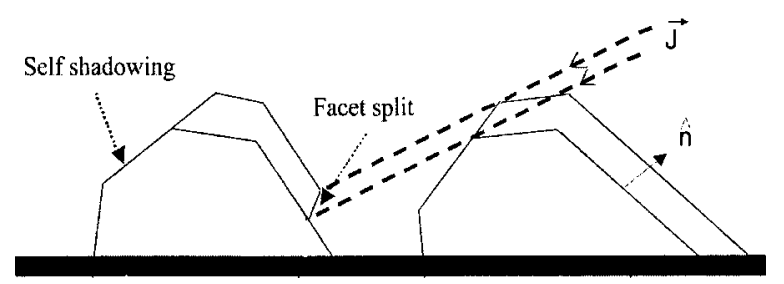

FIG. 2. Schematic illustration of the surface at two times indicating regions of no shadowing, self-shadowing (where one facet shadows an adjacent facet on the same crystallite), and facet splitting (where one crystallite shadows a facet on an adjacent crystallite). $\hat{n}$ is the facet normal and $\mathbf{J}$ is the deposition flux vector.

(2) one grain shadows or partially shadows facets on other grains.

When a facet is shadowed (i.e., it receives no flux), its velocity is set to zero. This changes the velocity of the vertices bounding the shadowed facet and, hence, modifies the evolution of the microstructure. A section of the surface is shadowed if it lies below a line parallel to the incident flux drawn from any of the other vertices further to the right than itself, assuming that the flux comes from the top right (see Fig. 2). If a segment of a facet is shadowed, a new vertex is introduced at the point that separates the segment with zero velocity from that with finite velocity.

The crystal symmetry of all nuclei are fixed when the islands are initially assigned a random orientation and no evolution of the orientation of the individual grains occurs during growth. The angles between facet normals were chosen to be $\phi=2 \pi / n$ and the included angles between facets themselves were $\theta=\pi-\phi=\pi(1-2 / n)$, where $n$ is an integer that describes the crystal symmetry. While 3, 4, and 6 are the only possible values of $n$ based upon space filling conditions, we treat $n$ as a general parameter here for completeness and also consider additional values of $n$. Some simulations were performed such that adjacent facets had different sticking coefficients, $S_{c}$. The sticking coefficient $S_{c}$ is the probability that an atom, incident from the vapor, will react with or adsorb onto the surface and become incorporated into the film. $S_{c}$ commonly varies from unity to less than $10^{-3}$ (Ref. 23) and varies with surface crystallography. In cases where all facets do not have $S_{C}=1$, the net growth rate of any facet (i) is adjusted, such that

$$
v_{i}=S_{C i}\left(\hat{n}_{i} \cdot \mathbf{J}\right)
$$

Since we only consider two types of facets, we arbitrarily assign the sticking coefficient of one to be unity and vary the value of $S_{c}$ of the other. (Note: this approach only works for even values of the symmetry factor $n$.) Except as noted below, we examine the microstructure and texture development for the case where all surfaces have the same sticking coefficient.

In order to obtain statistically meaningful results, a large number (1000) of nuclei are employed in each simulation. The simulation data reported below were averaged over 10 simulations to improve statistics. All length scales are normalized by the mean spacing between the nuclei at the beginning of the simulations, $d_{0}$.
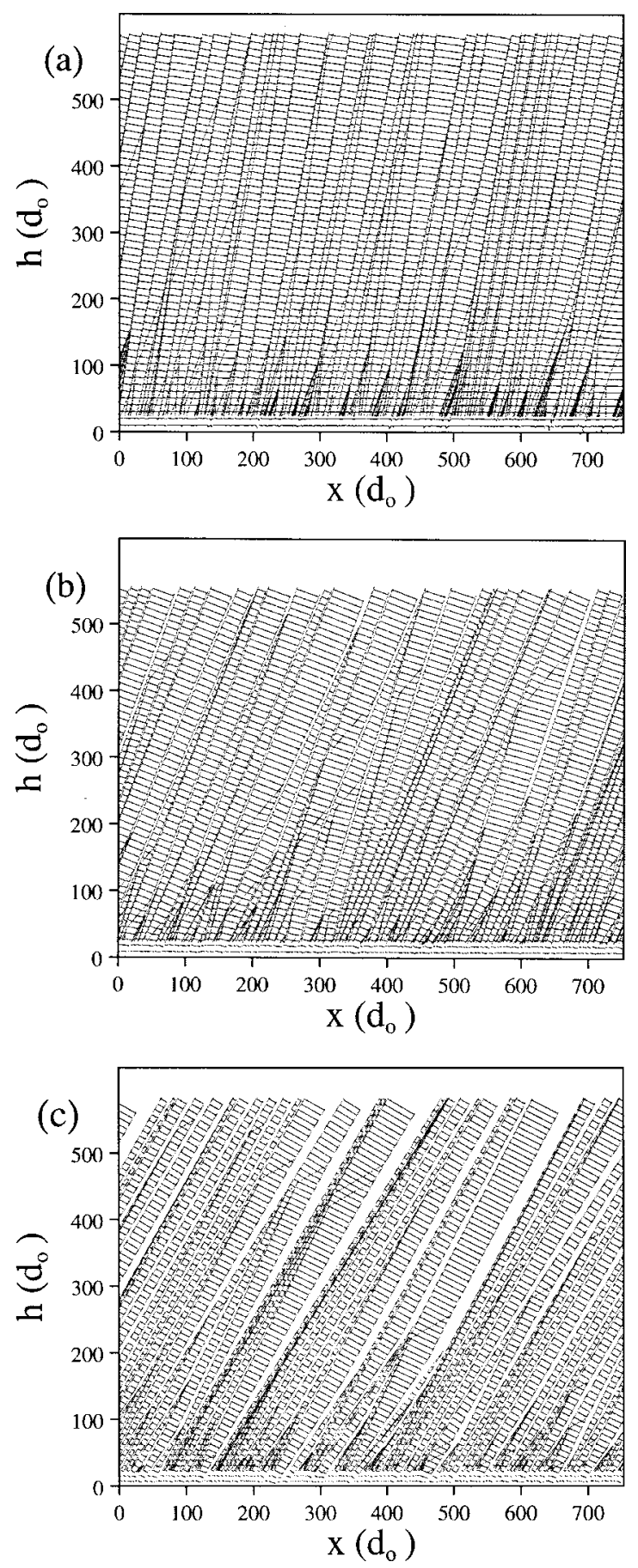

FIG. 3. The microstructure of polycrystalline films with $\phi=\pi / 2(n=4)$, $S_{c}=1$ deposited with (a) $\alpha=20^{\circ}$, (b) $\alpha=40^{\circ}$, and (c) $\alpha=60^{\circ}$. The regular set of lines indicate the position of the surface at regular intervals of time. The lines that intersect this regular set of lines correspond to grain boundaries and free surfaces. The grain boundaries are not shown below $h$ $=20 d_{o}$, where the number density of grain boundaries is extremely large. All lengths are in units of the mean spacing between the original crystalline nuclei on the substrate $\left(d_{0}\right)$.

\section{MICROSTRUCTURE DEVELOPMENT}

Figure 3 shows the temporal evolution of the microstructure and morphology of films deposited at several incident flux angles $\left(\alpha=20^{\circ}, 40^{\circ}, 60^{\circ}\right)$, as described in the previous section for the case of $\phi=\pi / 2$ (i.e., $n=4)$ and $S_{c}=1$. In the $\alpha=20^{\circ}$ [Fig. 3(a)] case, the grains are columnar (i.e., the 
grains at the top surface extend down to the substrate) and the grains are tilted towards the direction of the incident flux (however, $\beta<\alpha$ ) and the grain size coarsens as the film thickens. The increase in grain size with thickness is indicative of the competition between neighboring grains during growth, where the facets with larger velocity [Eq. (4)] increasingly shield their neighbors from the flux, eventually leading to grain pinch-off. Upon close examination, we see that true grain boundaries are rare and most grains are separated by very narrow, columnar voids. Although the number density of voids is high, the film is near full density (the voids are very narrow compared with the mean column width). Increasing the deposition angle to $\alpha=40^{\circ}$ [Fig. 3(b)] leads to notable changes in the microstructure. In this case, the grain size coarsens more rapidly and the grains are tilted more than for $\alpha=20^{\circ}$. This is in line with experimental observations $^{8}$ that show that the higher the deposition angle, the greater the angle by which the columns tilt away from the substrate normal. We also observe that true grain boundaries are rare, and individual grains are separated by readily visible voids that are considerably wider than in films grown at $\alpha=20^{\circ}$.

Figure 3(c) shows a microstructure grown with $\alpha$ $=60^{\circ}$. In this case, the column angle $(\beta)$ is larger and the film is even more porous than at the smaller deposition angles. Additionally, the mean void size is larger and the mean grain size is smaller than at smaller $\alpha$. There is a general trend toward increasingly fibrous grain structures with increasing $\alpha$, with voids that extend all the way from substrate to surface. These general morphological features are in agreement with a wide range of experiment results (see e.g., Refs. 5, 13).

The effect of changing crystal structure, as represented by changes in the facet angle, on the film microstructure and morphology can be seen by comparing Figs. 3 and 4. The simulations shown in Fig. 4 were performed for $\phi=\pi / 4$ ( $n$ $=8$ ) and $S_{c}=1$. Figure 4(a) shows the microstructure evolution for $\alpha=20^{\circ}$. In this case, the film is of full density, i.e., there are no voids. The grain boundaries are nearly parallel to each other and are oriented parallel to the deposition flux $(\beta \approx \alpha)$. Parallel grain boundaries imply no evolution of the grain size with film thickness, which is consistent with the microstructure in Fig. 4(a) after some small thickness. We observe similar behavior for deposition at $\alpha=40^{\circ}$ [Fig. 4(b)]. On the other hand, increasing the incident flux to $\alpha$ $=60^{\circ}$ results in a remarkably different microstructure than seen at lower $\alpha$. In this case, the microstructure is more similar to those in Fig. 3, $\phi=\pi / 2$. In the $\alpha=60^{\circ}$ case, the grain size obviously increases with thickness, not all boundaries are parallel to each other, voids between grains are frequent and $\beta<\alpha$. This suggests a link between the crystallography of the film material and the effectiveness of shadowing. We return to this point below.

\section{COLUMN ORIENTATION}

The variation of the mean column orientation $(\beta)$ with the angle of the incident vapor flux $(\alpha)$ is shown in Fig. 5. The column orientation is defined as the average angle the
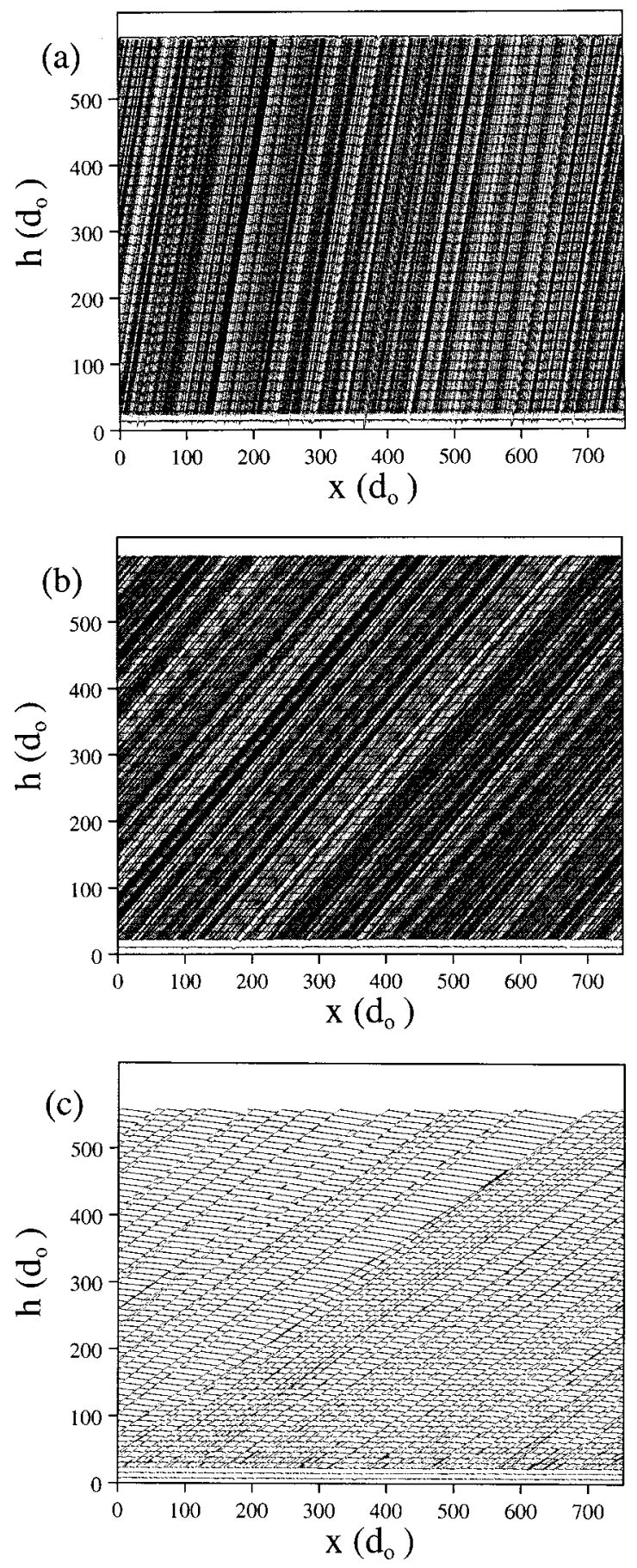

FIG. 4. The microstructure of polycrystalline films with $\phi=\pi / 4(n=8)$, $S_{c}=1$ deposited with (a) $\alpha=20^{\circ}$, (b) $\alpha=40^{\circ}$, and (c) $\alpha=60^{\circ}$.

grain boundaries or the side surfaces of the grains make with respect to the substrate normal. The simulation data in Fig. $5(\mathrm{a})$, where $\phi=\pi / 2(n=4)$ and $S_{c}=1$, shows that except for $\alpha=0^{\circ}, \beta<\alpha$. Based upon the data in Fig. 5(a), we can compare the predictions of the widely used tangent law [Eq. (1)] description of the column orientation versus angle of deposition with the $\phi=\pi / 2$ and $S_{c}=1$ simulation data. The agreement between the data and the tangent law is reasonable at low values of $\alpha$. As in most experiments, we find that above $60^{\circ}$ the deviations from the tangent law form becomes significant. Hara et $\mathrm{al}^{24}$ speculated that the cause of this de- 

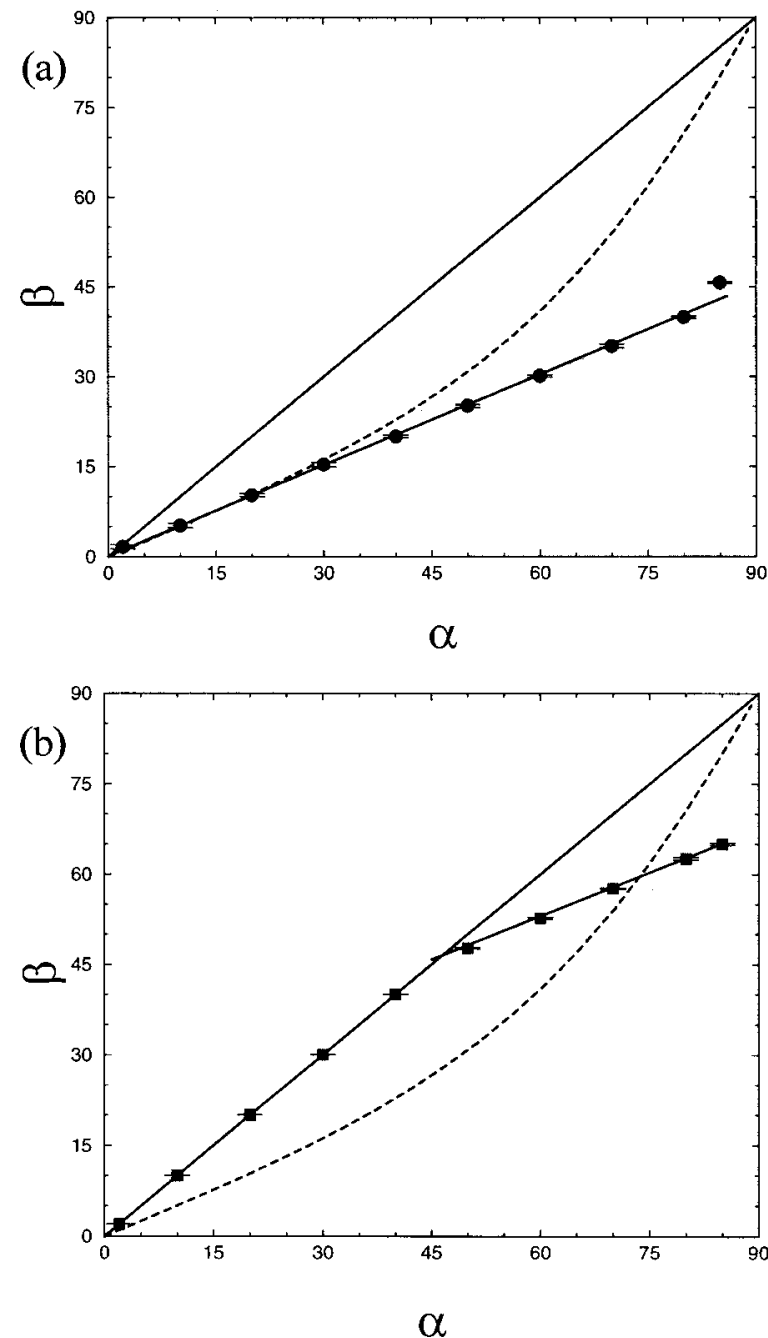

FIG. 5. (a) Column orientation $\beta$ vs vapor incidence angle $\alpha$ for $\phi=\pi / 2$ $(n=4), S_{c}=1$. The dashed line corresponds to the empirical tangent law [Eq. (1)]. Two straight lines are also shown corresponding to $\beta=\alpha$ and $\beta$ $=\alpha / 2$. (b) As in (a) for $\phi=\pi / 4(n=8), S_{c}=1$. The two straight lines here have slopes of 1 and $1 / 2$.

viation is directional diffusion, which arises because of the component of the momentum of the incident vapor flux parallel to the substrate. However, since we observe deviations from the tangent law similar to that seen in experiment and because the deposition flux in our simulations does not carry momentum, we can rule out that parallel momentum as the dominant cause for such deviations from the empirical tangent law.

While the tangent law does fit the data at small $\alpha$, the data appears to be better fit by a simple straight line of the form, $\beta=\alpha / 2$. The only deviations from this line occur at extremely high angle (i.e., $\alpha=85^{\circ}$ ). The agreement of the linear fit and the tangent law prediction at low angle is not surprising since an expansion of the tangent law [Eq. (1)] for small angle predicts $\beta=\alpha / 2$. On the other hand, the predictions of Lichter et al. ${ }^{10}$ [Eq. (2)] and Tait et al. ${ }^{11}$ suggest that $\beta=2 \alpha / 3$ and $\beta=\alpha$, respectively, at small $\alpha$, in clear disagreement with the simulation results. We return to these points below.
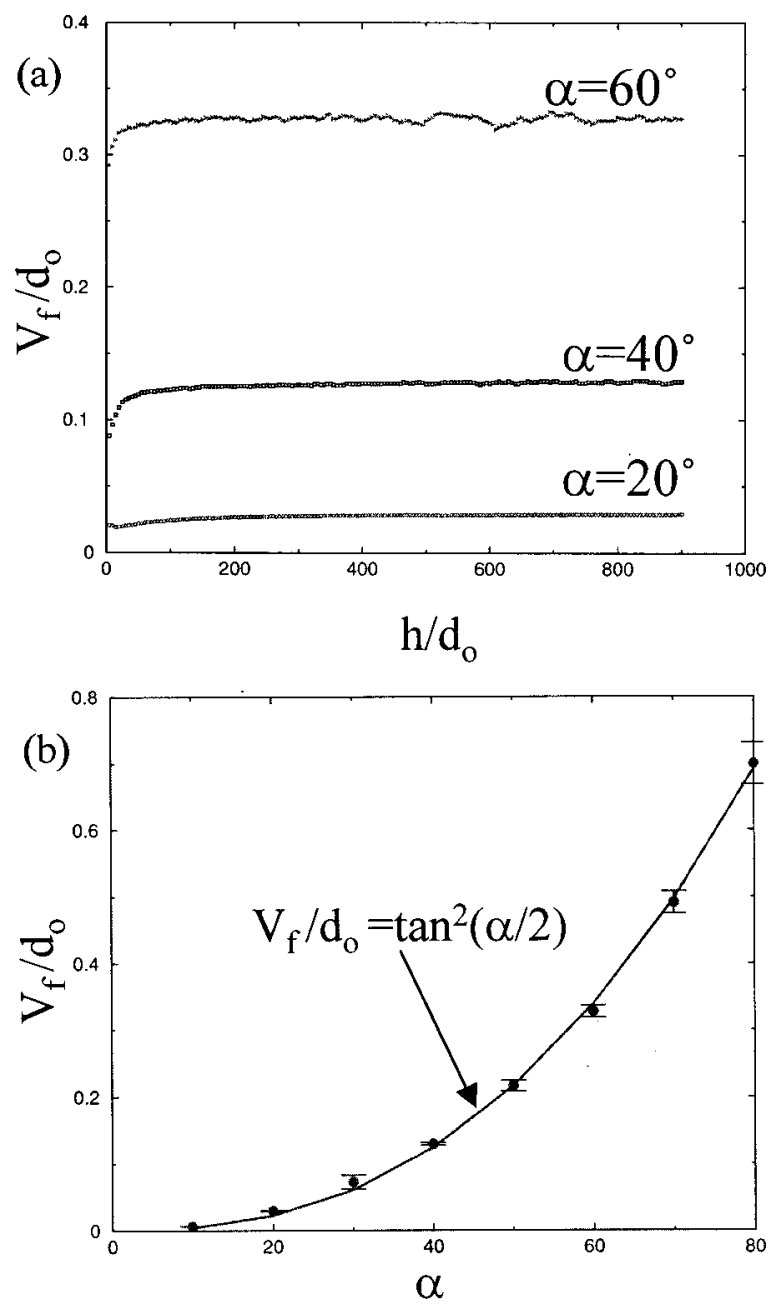

FIG. 6. (a) The total void fraction $V_{f}$ vs the film thickness $h$ for different deposition angles for $\phi=\pi / 2, S_{c}=1$. (b) As in (a) for $\phi=\pi / 2, S_{c}=1$. The predicted functional form of this data $V_{f}=\tan ^{2}(\alpha / 2)$ is also shown.

Figure 5(b) shows the column orientation versus incident angle for $\phi=\pi / 4(n=8)$ and $S_{c}=1$. We observe that for $0^{\circ} \leqslant \alpha \leqslant 45^{\circ}, \beta=\alpha$, as seen in the microstructures in Fig. 4 . This is in agreement with the predictions of Tait et al. ${ }^{11}$ at small $\alpha$, but in disagreement with the tangent law and the Lichter et al. ${ }^{10}$ prediction. However, for $\alpha \geqslant 45^{\circ}$, the column angle increasingly deviates from the $\beta=\alpha$ line with increasing $\alpha$. Examination of the data above $\alpha=45^{\circ}$ shows that in this range $\beta$ is a linear function of $\alpha$ [see the line in Fig. $5(\mathrm{~b})]$. Clearly the data for the $\phi=\pi / 4$ and $\phi=\pi / 2$ simulations are fundamentally different. The break in $\beta$ vs $\alpha$ observed in the $\phi=\pi / 4$ case does not occur in the $\phi=\pi / 2$ case. However, the slope of the $\alpha$ vs $\beta$ line in the $\phi=\pi / 2$ case matches that for the $\phi=\pi / 4$ case at $\alpha \geqslant 45^{\circ}$. We revisit this observation below.

\section{VOID, COLUMN, AND GRAIN SIZES}

The microstructures seen in Figs. 3 and 4 (for $\alpha>45^{\circ}$ ) all exhibit some degree of porosity. Figure 6(a) shows the evolution of the porosity (void fraction at a fixed height), $V_{f}$, with film thickness for $\phi=\pi / 2$ and $S_{c}=1$ for three different deposition angles, $\alpha$. Following a short transient the porosity 


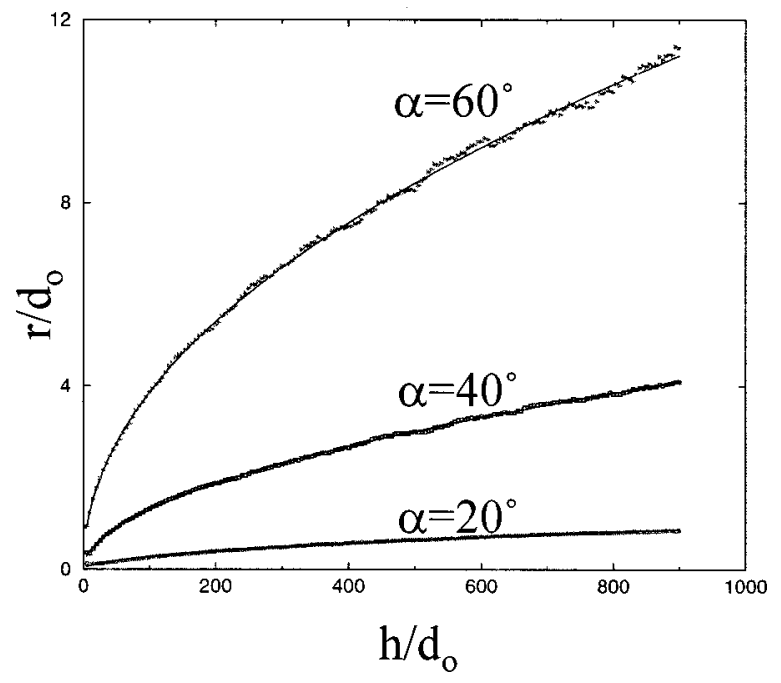

FIG. 7. The mean void size, $r / d_{0}$, vs the film thickness, $h / d_{0}, f$ for $\phi$ $=\pi / 2, S_{c}=1$. The solid lines are fits to $r=C d^{1 / 2}$, where $C$ is a constant.

asymptotes to a fixed, $\alpha$-dependent value. The variation of the asymptotic porosity with $\alpha$ is small at small $\alpha$ and then increases nearly quadratically with increasing $\alpha$ [see Fig. 6(b)]. The microstructures also show that the mean void size, $r$, increases with increasing thickness, $h$. This is quantified in Fig. 7 for $\phi=\pi / 2$ and $S_{c}=1$ for three deposition angles, $\alpha$. These data confirm that the mean void size does indeed increase with increasing thickness at a rate that increases with increasing deposition angle. A power law fit of the form $r$ $=A h^{\gamma}$ shows $\gamma=0.5 \pm 0.1$, where $A$ is a constant.

Since the porosity is independent of thickness at large thicknesses and the pore size increases with thickness, the data in Figs. 6 and 7 imply that the number of voids must be decreasing with increasing thickness. If this were true, the mean column size (measured parallel to the substrate) must increase with increasing thickness at the same rate that the void size grows. Figure 8, where we plot column size $D_{c}$ vs film thickness, confirms this deduction. A power law fit of the form $D_{c}=b h^{\delta}$ shows $\delta=0.5 \pm 0.1$, where $B$ is a constant.

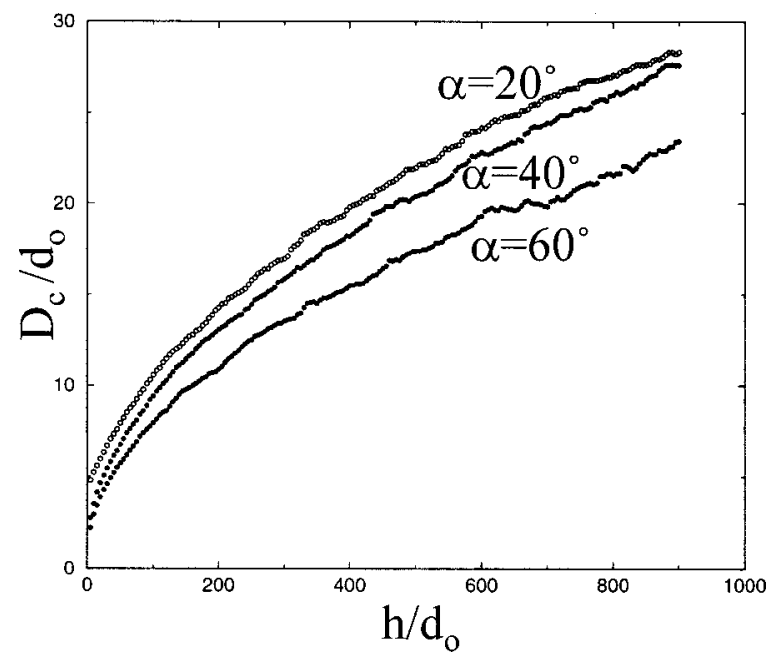

FIG. 8. The mean column size, $D_{c}$, vs the film thickness, $h / d_{0}$, for $\phi$ $=\pi / 2, S_{c}=1$.

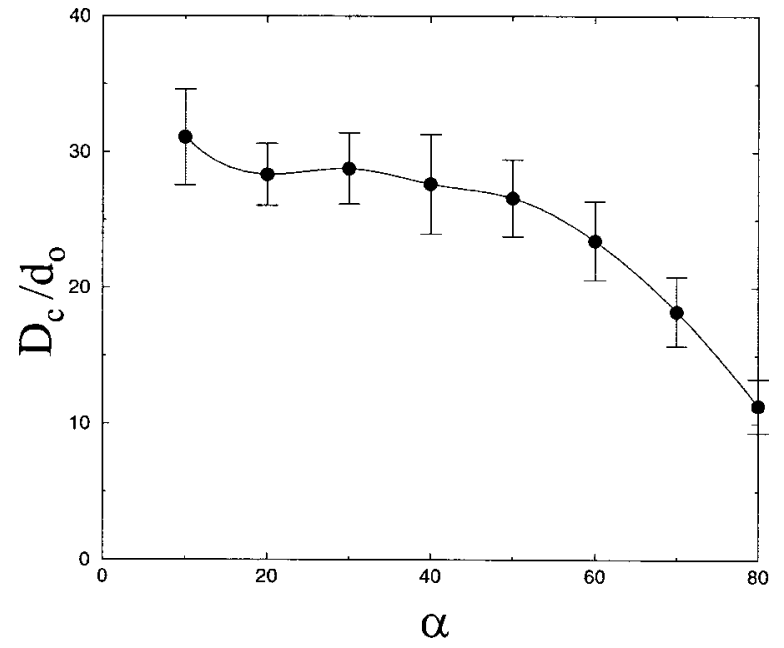

FIG. 9. The mean column size, $D_{c}$, vs deposition angle, $\alpha$, for $\phi=\pi / 2$, $S_{c}=1$ at $h / d_{0}=800$.

While the mean column size increases with increasing thickness, the rate of increase is greater for smaller deposition angles. This is the opposite behavior from that seen for the mean void size [Fig. 6(a)], as must be the case since the steady-state porosity is larger for larger deposition angles. Figure 9 shows that although the mean column size is insensitive to $\alpha$ for small $\alpha$ it decays rapidly with $\alpha$ at large $\alpha$ (all at fixed film thickness). This is consistent with the discrete particle simulation data of Tait et al. ${ }^{11}$

The microstructures in Figs. 3 and 4 clearly show that at least some of the columns consist of single grains (i.e., single crystal columns). Unfortunately, because many voids are very narrow, it is not obvious whether most of the columns are single crystals or are polycrystalline. In order to clarify this point, we measured the mean number of grains per column $N_{c}$ as a function of film thickness for $\phi=\pi / 2$ and $S_{c}$ $=1$ for three deposition angles, $\alpha$ (see Fig. 10). Following an initial transient where the columns are polycrystalline, the

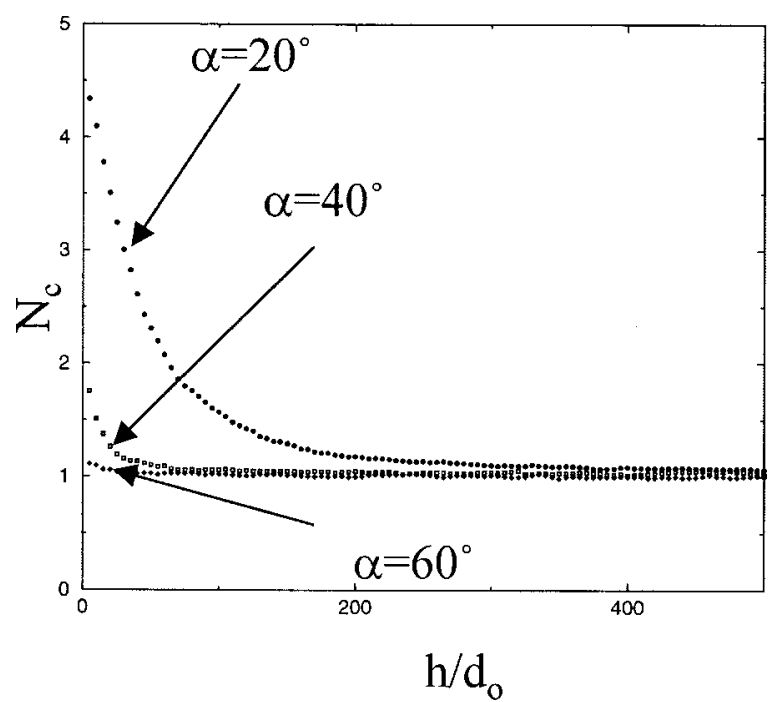

FIG. 10. The number of grains per column, $N_{c}$, vs film thickness, $h / d_{0}$, for $\phi=\pi / 2, S_{c}=1$. 


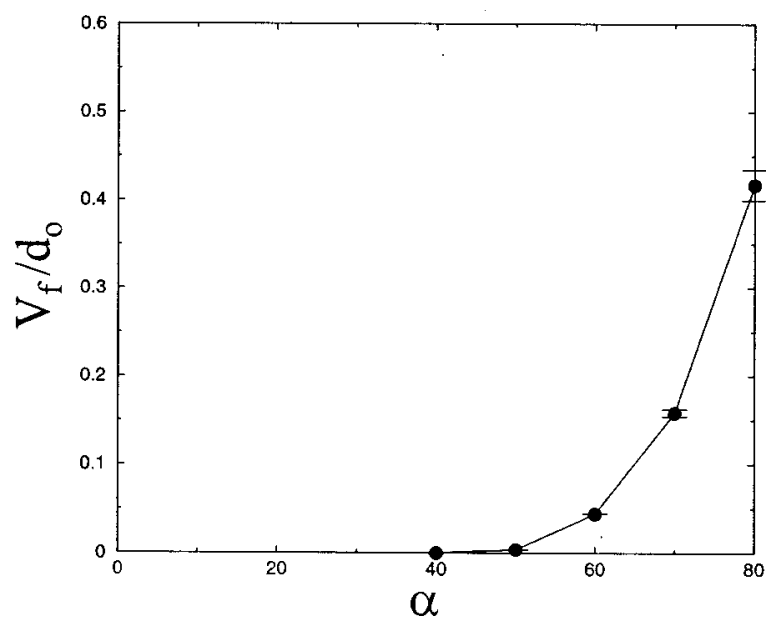

FIG. 11. Void fraction, $V_{f}$, vs deposition angle, $\alpha$, for $\phi=\pi / 4, S_{c}=1$.

number of grains per columns rapidly asymptote to unity, implying that single crystal columns are the rule.

The microstructures obtained under low angle deposition conditions $\left(\alpha<45^{\circ}\right)$ for $\phi=\pi / 4$ and $S_{c}=1$ appear to be fundamentally different from those obtained at high angles $\left(\alpha>45^{\circ}\right)$ or those obtained for depositions with $\phi=\pi / 2$ and $S_{c}=1$ [cf. Figs. 4(a) and 4(b) with Figs. 3 and 4(c)]. In the low angle, $\phi=\pi / 4$ case, the grain sizes and void sizes do not significantly coarsen during growth and the films are nearly full density (see Fig. 11). Above $45^{\circ}$, however, the grain size, pore size, and porosity all follow the same trends observed in the $\phi=\pi / 2$ films (see e.g., Fig. 11).

\section{CRYSTALLOGRAPHIC TEXTURE}

We now examine the evolution of the crystallographic texture during film growth. By crystallographic texture, we imply the distribution of the orientations of the crystallographic axes throughout the material. In particular, we focus on the distribution of those directions that are perpendicular to the facets. For example, for the $\phi=\pi / 2$ films, we examine the distribution of the $\langle 01\rangle$ axes with respect to the substrate normal, $\theta$. Since the crystals posses a rotation axis, we only consider the smallest (positive) angle that one of the $\langle 01\rangle$ directions make with respect to the normal. For $\phi=\pi / 2$, this implies an angular range $0 \leqslant \theta<\pi / 2$ and for $\phi=\pi / 4,0 \leqslant \theta$ $<\pi / 4$.

The probability distribution of the grain orientations, $P(\theta, h)$, as a function of both orientation $\theta$ and film thickness $h$ is shown in Fig. 12 for $\phi=\pi / 2$ and $S_{c}=1$ for three deposition angles, $\alpha$. Since each simulation was started with randomly oriented nuclei on the substrate, the initial distribution of crystal orientations $P(\theta, 0)$ is flat. The orientation distribution develops a peak at small thicknesses that sharpens with increasing growth and then asymptotes. The peak position, $\theta_{\max }$, increases from $10.3^{\circ}$ at $\alpha=20^{\circ}$ [Fig. 12(a)] to $19.8^{\circ}$ at $\alpha=40^{\circ}$ [Fig. 12(b)] to $30.2^{\circ}$ at $\alpha=60^{\circ}$ [Fig. 12(c)]. There is a general trend of $\theta_{\max }$ increasing with increasing $\alpha$. This demonstrates that shadowing strongly modifies crystallographic texture.
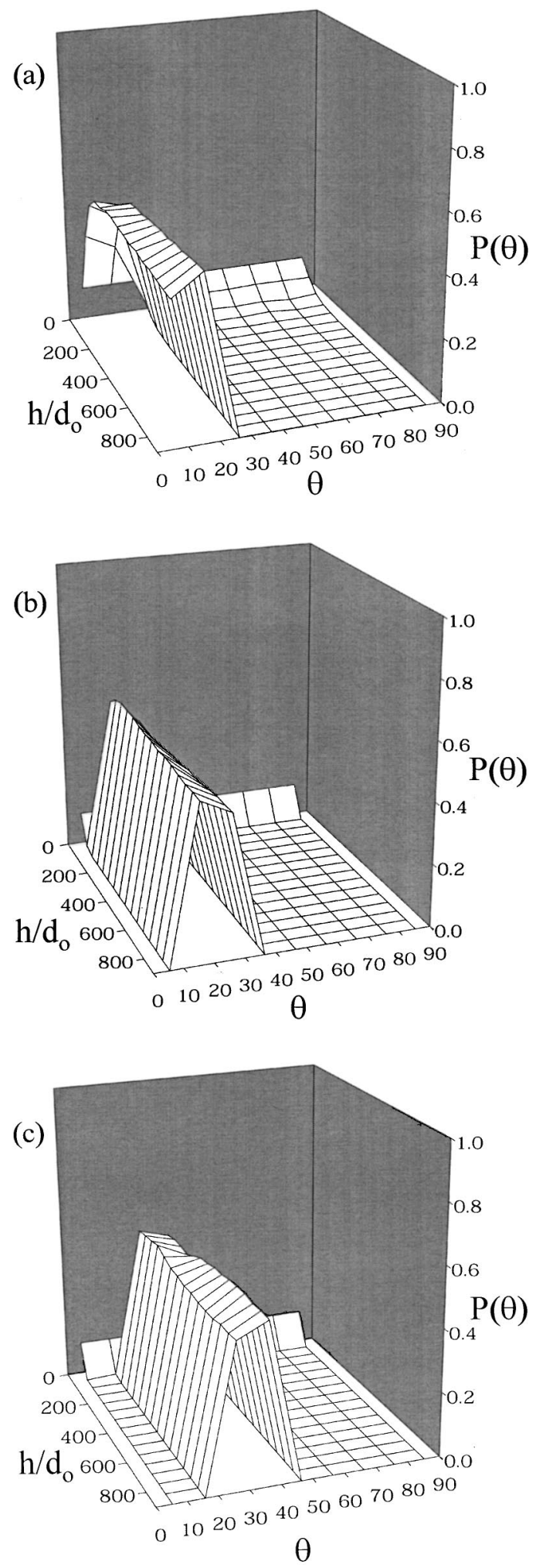

FIG. 12. Orientation distribution function $P(\theta)$ vs film thickness, $h / d_{0}$ for $\phi=\pi / 4, S_{c}=1$ for films deposited at (a) $\alpha=20^{\circ}$, (b) $\alpha=40^{\circ}$, and (c) $\alpha$ $=60^{\circ}$.

Since we defined the crystallographic texture in terms of the angle between the facet normal and the substrate normal, $\theta_{\max }$ can be thought of as also describing the orientation of the most common facet normals. These values of $\theta_{\max }$ are consistent with the facet normals on the upper surfaces of the 

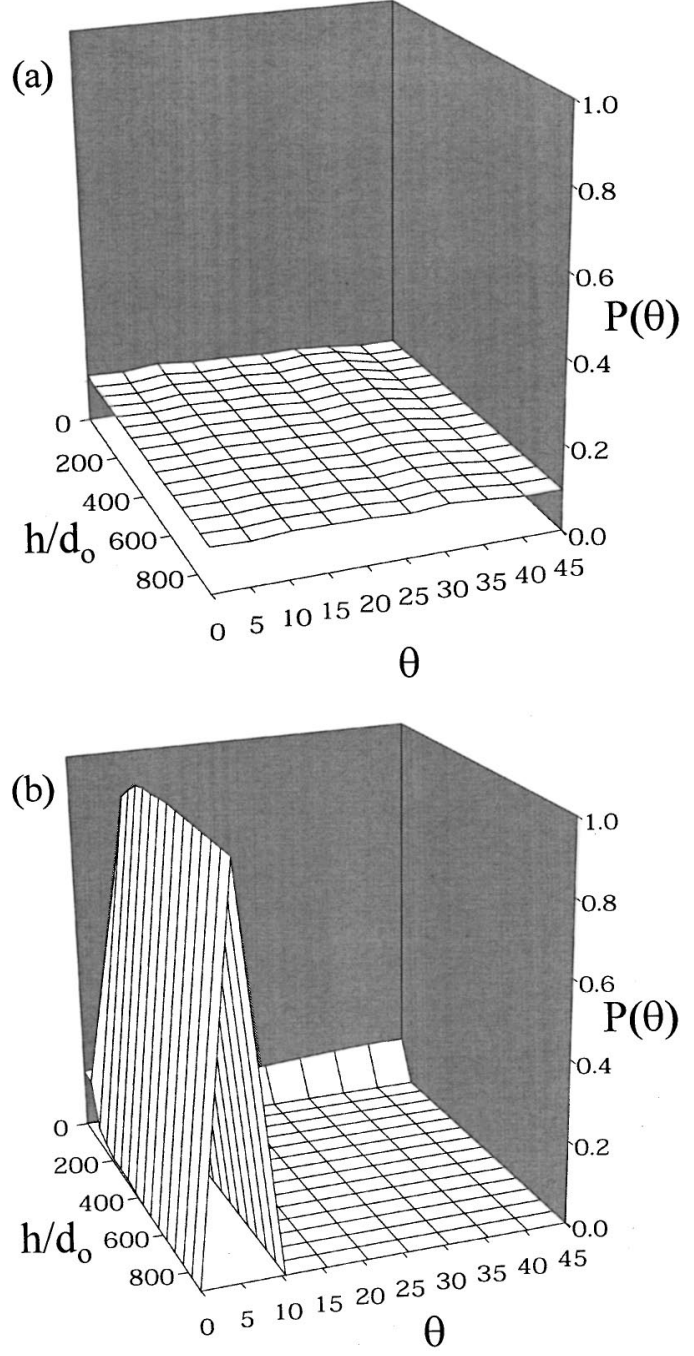

FIG. 13. Orientation distribution function $P(\theta)$ vs film thickness, $h / d_{0}$ for $\phi=\pi / 8, S_{c}=1$ for films deposited at (a) $\alpha=20^{\circ}$, (b) $\alpha=40^{\circ}$, and (c) $\alpha$ $=60^{\circ}$.

grains in the microstructures in Fig. 3, which show an increase in angle with increasing $\alpha$. Examination of the microstructures and facets in Fig. 3 demonstrate that the relative orientations of the facets at the growing surface and the surfaces on the sides of the columns have the same orientation relationship as the normal crystal facets themselves, i.e., $\phi$ $=\pi / 2$ in the present case. This implies that there should be some relationship between $\beta$ and $\theta_{\max }$. In fact, comparison of Figs. 5(a) and 12 suggest that $\theta_{\max }=\beta$.

Just as the microstructures for the $\phi=\pi / 4$ case and $\alpha$ $<45^{\circ}$ are markedly different from those at either $\alpha>45^{\circ}$ or $\phi=\pi / 2$, the texture in these cases are also fundamentally different. Figure 13 shows $P(\theta, h)$ for $\phi=\pi / 4$ and $S_{c}=1$ for $\alpha=20^{\circ}$ and $60^{\circ}$. For $\alpha \leqslant 45^{\circ}$, the orientation distribution remains flat during the entire growth process. This indicates that the texture starts random and remains random. This is consistent with the lack of microstructural evolution (void or column size) during growth. On the other hand, for $\alpha$ $>45^{\circ}$, a peak in the distribution function forms at early times, sharpens as the film grows and then asymptotes, much as it does in the $\phi=\pi / 2$ case for all $\alpha$.

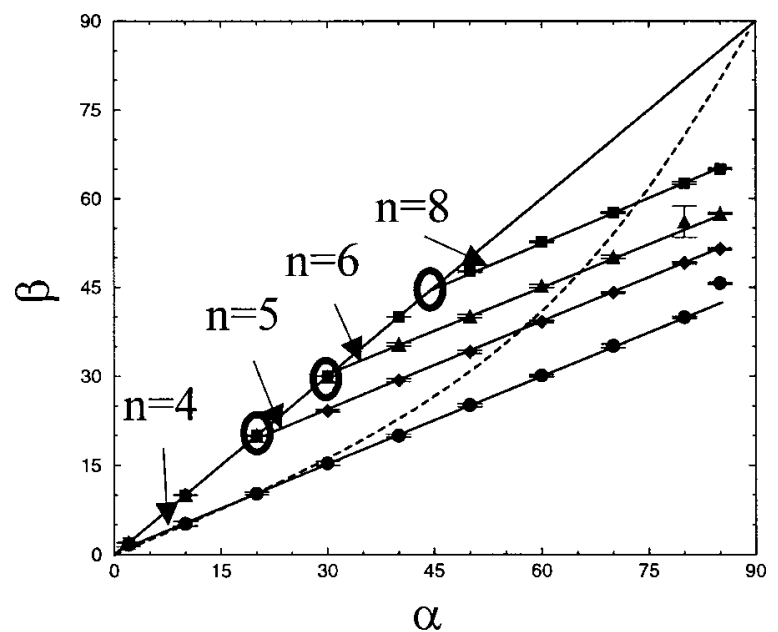

FIG. 14. Column orientation, $\beta$, vs vapor incidence angle, $\alpha$, for $S_{c}=1$ and different values of $\phi=2 \pi / n$. The circled regions indicate the values of $\alpha_{c}$ and the straight lines beyond $\alpha_{c}$ correspond to $\beta=\left(\alpha+\alpha_{c}\right) / 2$.

\section{EFFECTS OF CRYSTAL SYMMETRY}

Much of the microstructural and texture data presented above showed that the film structure that develops during growth is strongly influenced by the crystal structure. The relationship of the grown microstructures to the crystal structure is through the relative facet orientations as represented by the parameter $\phi$, which indicates the rotational symmetry of the crystal structure $(\phi=2 \pi / n$, where $n$ is the degree of the rotation axis, e.g., $n=4$ implies a fourfold symmetry and $\phi=\pi / 2$ ). One key area where the crystal structure enters is in the relationship between the column orientation and the deposition angle. In Fig. 14 we show $\beta$ vs $\alpha$ for the cases $\phi=2 \pi / 4,2 \pi / 5,2 \pi / 6$, and $2 \pi / 8$. We note that $n=5$ and $n$ $=8$ correspond to rotational symmetries that cannot occur in two dimensional systems, but we include them, nonetheless, to aid in discerning trends. The data for each $\phi$ falls on two lines. For the deposition angle less than a critical angle ( $\alpha$ $\leqslant \alpha_{c}$ ), the data is independent of $n$ (or $\phi$ ) and is well described by the relation $\beta=\alpha$. For $\alpha>\alpha_{c}$, the data for each $n$ or $\phi$ fall along a single line, $\beta=\left(\alpha_{c}+\alpha\right) / 2 . \alpha_{c}$ is a function of $\phi$ or $n$, such that the data for $\alpha>\alpha_{c}$ lie on parallel lines. Clearly, $\alpha_{c}$ decreases with increasing $n$. $\alpha_{c}$ is well described by

$$
\alpha_{c}=\frac{\pi}{2}\left(1-\frac{4}{n}\right) .
$$

The type of microstructure and texture data observed (see above) changes abruptly at $\alpha=\alpha_{c}$. For $\alpha<\alpha_{c}$, the grain size is extremely small and the film is unstructured, while for $\alpha \geqslant \alpha_{c}$, grains and voids growth with increasing thickness and a strong texture is formed. Unlike in the $n$ $=8(\phi=\pi / 4)$ case, there are no abrupt changes in the behavior of the $n=4(\phi=\pi / 2)$ microstructure or texture data simply because $\alpha_{c}(n=4)$ is identically zero, i.e., all $\alpha$ $\geqslant \alpha_{c}$. 


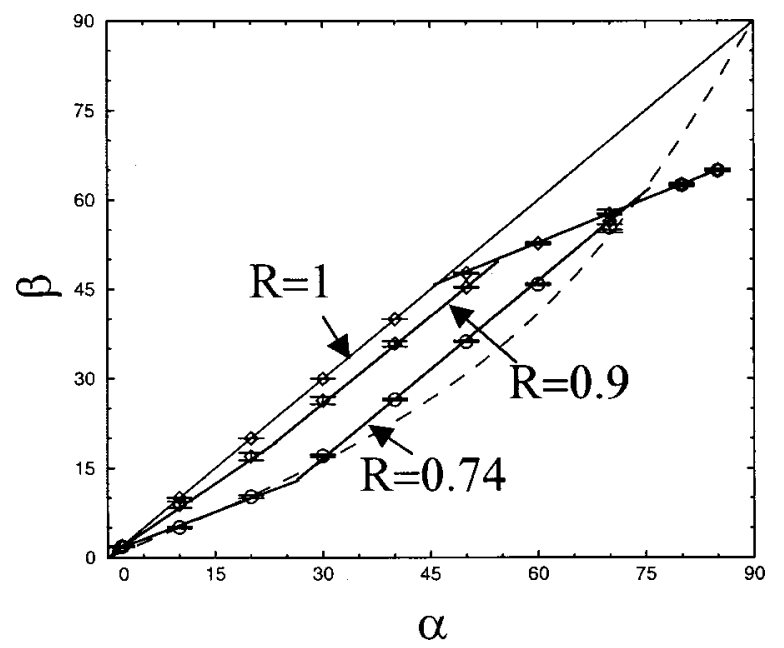

FIG. 15. Column orientation, $\beta$, vs deposition angle, $\alpha$, for $\phi=\pi / 4$ and varying $R$. Also shown are best linear fits and the tangent law.

\section{EFFECTS OF NONUNIFORM STICKING COEFFICIENTS}

The results of the last section demonstrated that the microstructure is sensitive to the relative orientations of neighboring facets, $\phi$. In this section, we investigate what happens if $\phi$ is fixed but the relative growth rates of neighboring facets differ. This can happen in situations where neighboring facets have different sticking coefficients $S_{c i}$ [see Eq. (5)]. We define the ratio of sticking coefficients of adjacent faces as $R=S_{c i} / S_{c i+1} \leqslant 1$, where the subscripts $i$ and $i+1$ label adjacent facets. By making $R \neq 1$, we imply that the crystal symmetry is lower by a factor of 2 compared with that the $R=1$ case at the same value of $n$ (i.e., an eightfold rotation axis reduces to a fourfold axis). Nonetheless, even when $R \neq 1$, the angle between adjacent facets remains fixed at $\phi=2 \pi / n$. Therefore, allowing sticking coefficients to differ from unity provides an intermediate case between an $n$-fold and ( $n / 2)$-fold situation.

Figure 15 shows $\beta$ vs $\alpha$ for the case of $\phi=\pi / 4$ and $R$ $=1,0.9$, and 0.74 . The data for $R=1$ is the same as that shown in Fig. 5(b); two straight line with a break at $\alpha=\alpha_{c}$ $=\pi / 4$. For $R<1$, the data shows three regimes: one for $\alpha$ $>\alpha_{c}$, where $\beta=\left(\alpha_{c}+\alpha\right) / 2$ and $\alpha_{c}$ is described by Eq. (6), an intermediate one where $\beta=\alpha+C_{1}$, where $C_{1}$ is a constant, and a small $\alpha$ regime where $B=C_{2} \alpha$ and $C_{2}<1$. The slope $\left(C_{2}\right)$ in this small $\alpha$ regime decreases with decreasing $R$.

\section{DISCUSSION AND CONCLUSIONS}

We have performed a series of simulations designed to clarify the effects of shadowing on the evolution of the film structure in the absence of other factors that are thought to be important in determining film microstructure such as surface diffusion and the discrete size of the deposition species. The simulations track the evolution of the two-dimensional, faceted growth front during the oblique deposition of vapor atoms to grow polycrystalline films. The simulations show that that even in the absence of these other physical effects, the resultant films exhibit a columnar microstructure, where the columns are typically single crystals. As the deposition angle increases, the microstructure becomes increasingly porous and the column tilt in the direction towards that of the deposition flux. Both of these observations are consistent with experiment ${ }^{25}$ and other simulations. ${ }^{16,26}$ Above a critical deposition angle, the mean void and grain sizes are proportional to each other; both scale quadratically with film thickness and, following an initial transient, the porosity is independent of film thickness. The dependence of the column orientation $\beta$ on the deposition angle $\alpha$ was found to be in poor agreement with the empirical tangent law, which reasonably describes a wide range of experimental observations. ${ }^{8}$ This suggests that while shadowing is a necessary ingredient to obtain tangent lawlike behavior, by itself, it is insufficient. This supports the notion that a finite deposition species size may be necessary to obtain such behavior. ${ }^{24}$ We also observed that the relationship between $\beta$ and $\alpha$, and the entire form of the microstructure, are sensitive to the symmetry of the underlying crystal in systems that exhibit faceting.

The relationship between the column $\beta$ and deposition $\alpha$ angles seen in the $(R=1)$ simulations shows two distinct regimes. For $\alpha<\alpha_{c}, \beta=\alpha$ and for $\alpha<\alpha_{c}, \beta=\left(\alpha+\alpha_{c}\right)$. It is easy to see where the transition in behavior occurs $\left(\alpha_{c}\right)$ in terms of the relative orientation of the facets and the deposition flux. Consider the growth of an $n$-sided polygon (corresponding to a grain with $\phi=2 \pi / n, R=1$ ) on a substrate in a flux of orientation $\alpha$ and ignore those facets that grow toward the substrate (as they disappear rapidly). As $\alpha$ increases from zero, it will reach a value such that one of the facets that used to receive flux, no longer receives any flux (i.e., the flux direction is tangent to the surface) and therefore stops growing. Simple geometry shows that this will occur first when $\alpha=(\pi / 2)(1-4 / n)$, where one must consider all possible rotations of the polygon. This is exactly the expression we found for $\alpha_{c}$ based on the simulation data [Eq. (6)]. Therefore, the critical value of $\alpha$ simply results from the abrupt change in how many facets receive flux and grow.

For $\alpha<\alpha_{c}$, the simulations show that $\beta=\alpha$. We can rationalize this result by considering the trajectories of the individual facet vertices in this case where shadowing does not occur $\left(\alpha=\alpha_{c}\right.$ is the onset of shadowing in faceted structures). Assuming each facet moves with the velocity given by Eq. (4), the slope of the trace of each vertex, $m_{v}$, as it moves in time is simply

$$
m_{v}=\tan \alpha .
$$

This relation is valid for all moving vertices, independent of the angle that the individual facets make with respect to the deposition flux. Hence, each vertex moves directly parallel to the deposition flux. This implies that the microstructure does not evolve at all during growth. This is consistent with the observed microstructures in Figs. 5(a) and 5(b) and the thickness dependence of the grain size, the column size, and texture [Fig. 12(a)]. If each vertex moves parallel to the deposition flux, the columns (which are obviously bounded by vertices) must also move with the same slope. Hence, $\tan \beta$ $=\tan \alpha$ or $\beta=\alpha$ for $\alpha<\alpha_{c}$, as observed in the simulations. We have not found a similar simple explanation to account 


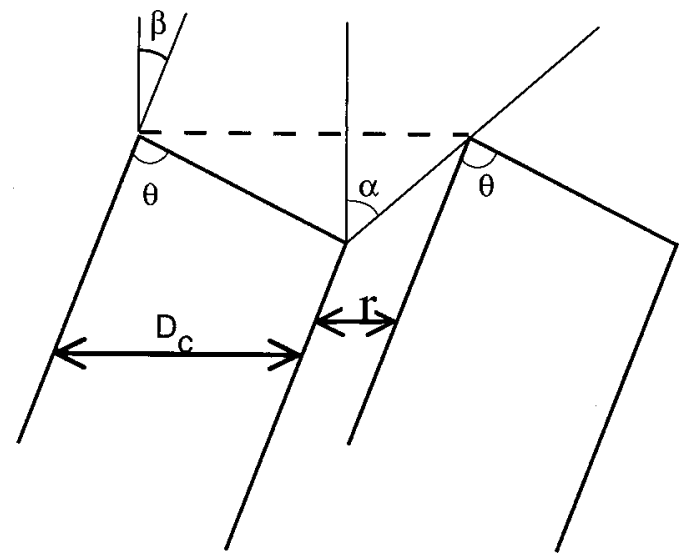

FIG. 16. Schematic illustration of one grain shadowing its neighbor. $r$ is the void size, $D_{c}$ is the column width, and $\theta=\pi-\phi$.

for the behavior of $\beta$ vs $\alpha$ for $\alpha \geqslant \alpha_{c}$ [i.e., the observed relationship $\left.\beta=\left(\alpha+\alpha_{c}\right) / 2\right]$. In the limit of nonfaceted films $(\phi \neq 0, n \neq \infty), \alpha_{c} \neq \pi / 2$ and, hence, $\beta=\alpha$. This is consistent with the conclusions reached by Tait et al. ${ }^{11}$ in the noshadowing limit, but not with experiment.

The simulation data shows that the void fraction $V_{f}$ (porosity) of the film increases with increasing $\alpha$. We can understand the relationship between the porosity and the deposition angle by considering how shadowing from a tilted column effects the flux received by a neighboring column, as shown in the schematic in Fig. 16. We can define the void fraction in terms of the column $\left(D_{c}\right)$ and void $(r)$ size as $V_{f}=r /\left(r+D_{c}\right)$. With this definition and the definitions in Fig. 16, simple geometry shows

$$
V_{f}=\frac{\cos (\beta-\theta) \sin (\alpha-\beta)}{\cos (\alpha) \sin (\theta)+\sin (\alpha-\beta) \sin (\beta)} .
$$

The simulation data (Fig. 15) demonstrate that for $\alpha \leqslant \alpha_{c}$, $\beta=\alpha$. Substituting $\beta=\alpha$ into Eq. (8) implies that the film is of full density, i.e., $V_{f}=0$. This is consistent with the simulation data in Fig. 11. As $\alpha$ tends to $\pi / 2$, the film density goes to zero $\left(V_{f}=1\right)$ since $\beta=\left(\alpha+\alpha_{c}\right) / 2$ for $\alpha>\alpha_{c}$ (recall that $\alpha_{c} \leqslant \pi / 2$ in all cases). For the case of $\phi=\pi / 2(n=4), \beta$ $=\alpha / 2$ [as observed from Fig. 5(a)] and Eq. (8) reduces to

$$
V_{f}=\tan ^{2}\left(\frac{\alpha}{2}\right) \text {. }
$$

Figure 6(b) shows a comparison between the prediction of Eq. (8) and the simulation data for $\phi=\pi / 2$. The agreement is clearly excellent with no adjustable parameters.

The simulation data showed that the mean void size (Fig. 7) and column size (Fig. 8) scaled with the film thickness (for $\alpha>\alpha_{c}$ ) as $h^{1 / 2}$. This parabolic growth behavior is similar to that seen in the growth of faceted films under other conditions. ${ }^{27}$ Consider the surfaces of one grain surrounded by two voids. In the general case, the two surfaces adjacent to the two voids will not be parallel and, hence, at some finite height they will hit each other, the intervening grain will disappear and two voids will coalesce. Similarly, if two columns are growing, but are not quite growing exactly parallel to each other, they will eventually impinge on each other, thereby pinching-off the intervening void. Clearly, the evolution of the void and column sizes is intimately related with the evolution of the texture (which controls how parallel the growth directions are). An analysis of the evolution of texture and grain size was performed based upon this relationship. ${ }^{20,28}$ This relationship shows that as the texture sharpens, the columns become increasingly parallel and the impingement of columns becomes less frequent, resulting in $D_{c}=A h^{1 / 2}$ (where $A$ is a constant), in agreement with the present simulations. A simpler approach is simply to realize that a column is pinched off when a column hits another column. If $N$ is the number of columns in a finite width film, this implies that $d N / d t=B N$ (where $B$ is a constant). Since $N$ is inversely proportional to the scale of the microstructure (column or void width), this also implies that $D_{c}=A h^{1 / 2}$. The fact that both the mean column and void width grow as $h^{1 / 2}$ implies that the film density is independent of thickness, as discussed above.

\section{ACKNOWLEDGMENTS}

The authors would like to gratefully acknowledge the support of the U.S. Department of Energy through Grant No. DE-FG02-99ER45797.

${ }^{1}$ T. G. Knorr and R. W. Hoffmann, Phys. Rev. 113, 1039 (1959).

${ }^{2}$ D. O. Smith, J. Appl. Phys. 30, 264S (1959).

${ }^{3}$ A. G. Dirks and H. J. Leamy, Thin Solid Films 47, 219 (1977).

${ }^{4}$ K. Starbova, V. Mankov, J. Dikova et al., Vacuum 53, 441 (1999).

${ }^{5}$ K. Starbova, J. Dikova, and N. Starbov, J. Non-Cryst. Solids 210, 261 (1997).

${ }^{6}$ A. Hagemeyer, H. J. Richter, H. Hibst et al., Thin Solid Films 230, 199 (1993).

${ }^{7}$ O. P. Karpenko, J. C. Billelo, and S. M. Yalisove, J. Appl. Phys. 82, 1397 (1997)

${ }^{8}$ J. M. Nieuwenhuizen and H. B. Haanstra, Philips Tech. Rev. 27, 87 (1966).

${ }^{9}$ N. G. Nakhodin and A. I. Shaldervan, Thin Solid Films 10, 109 (1972).

${ }^{10}$ S. Lichter and J. Chen, Phys. Rev. Lett. 56, 1986 (1986).

${ }^{11}$ R. N. Tait, T. Smy, and M. J. Brett, Thin Solid Films 226, 196 (1993).

${ }^{12}$ D. K. Pandya, A. C. Rastogi, and K. L. Chopra, J. Appl. Phys. 46, 2966 (1975).

${ }^{13}$ D. Vick, L. J. Friedrich, S. K. Dew, M. J. Brett, K. Robbie, M. Seto, and T. Smy, Thin Solid Films 339, 88 (1999).

${ }^{14}$ G. S. Bales and A. Zangwill, J. Vac. Sci. Technol. A 9, 145 (1991).

${ }^{15}$ K.-H. Muller, J. Appl. Phys. 58, 2573 (1985).

${ }^{16}$ S. Levine and P. Clancy, Modell. Simul. Mater. Sci. Eng. 8, 751 (2000).,

${ }^{17}$ L. Dong, R. W. Smith, and D. J. Srolovitz, J. Appl. Phys. 80, 5682 (1996).

${ }^{18}$ P. Meakin and J. Krug, Europhys. Lett. 11, 7 (1990).

${ }^{19}$ Paritosh and D. J. Srolovitz, J. Appl. Phys. 894857 (2001).,

${ }^{20}$ Paritosh, D. J. Srolovitz, C. Battaile, X. Li, and J. E. Butler, Acta Mater. 47, 2269 (1999).

${ }^{21}$ A. van der Drift, Philips Res. Rep. 22, 267 (1967).

${ }^{22}$ S. Barrat, P. Pigeat, and E. Bauer-Grosse, Diamond Relat. Mater. 5, 276 (1996).

${ }^{23}$ Donald L. Smith, Thin-Film Deposition (McGraw-Hill, New York, 1995).

${ }^{24}$ L. Abelman and C. Lodder, Thin Solid Films 305, 1 (1997).

${ }^{25}$ H. J. Leamy, G. H. Gilmer, and A. G. Dirks, in Current Topics in Material Science, edited by E. Kaldis (North-Holland, New York, 1980), Vol. 6, p. 309.

${ }^{26}$ T. Smy, K. L. Westra, and M. J. Brett, IEEE Electron Device Lett. 10, 19 (1989).

${ }^{27}$ Y. von Kaenel, J. Stiegler, E. Blank, O. Chauvet, C. Hellwig, and K. Plamann, Phys. Status Solidi A 154, 219 (1996).

${ }^{28}$ J. M. Thijssen, Phys. Rev. B 51, 1985 (1995). 